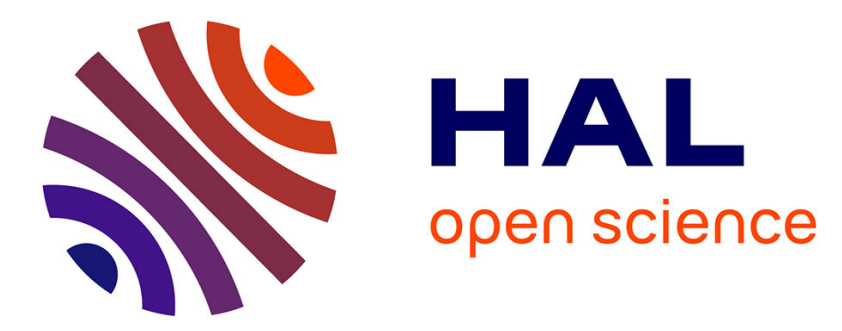

\title{
Organisation et accountabilitydes échanges langagiers lors d'auditions judiciaires
}

\author{
Esther González-Martínez
}

\section{To cite this version:}

Esther González-Martínez. Organisation et accountabilitydes échanges langagiers lors d'auditions judiciaires. Réseaux: communication, technologie, société, 2005, 129-130 (1), 10.3917/res.129.0209 . hal-01859244

\author{
HAL Id: hal-01859244 \\ https://hal.science/hal-01859244
}

Submitted on 21 Aug 2018

HAL is a multi-disciplinary open access archive for the deposit and dissemination of scientific research documents, whether they are published or not. The documents may come from teaching and research institutions in France or abroad, or from public or private research centers.
L'archive ouverte pluridisciplinaire HAL, est destinée au dépôt et à la diffusion de documents scientifiques de niveau recherche, publiés ou non, émanant des établissements d'enseignement et de recherche français ou étrangers, des laboratoires publics ou privés. 


\title{
ORGANISATION ET ACCOUNTABILITYDES ÉCHANGES LANGAGIERS LORS D'AUDITIONS JUDICIAIRES
}

\author{
Esther González Martínez \\ La Découverte | Réseaux
}

2005/1 - n० 129-130

pages 209 à 241

ISSN 0751-7971

Article disponible en ligne à l'adresse:

http://www.cairn.info/revue-reseaux-2005-1-page-209.htm

Pour citer cet article :

González Martínez Esther, « Organisation et accountabilitydes échanges langagiers lors d'auditions judiciaires », Réseaux, 2005/1 n¹29-130, p. 209-241. DOI : 10.3917/res.129.0209

Distribution électronique Cairn.info pour La Découverte.

(c) La Découverte. Tous droits réservés pour tous pays.

La reproduction ou représentation de cet article, notamment par photocopie, n'est autorisée que dans les limites des conditions générales d'utilisation du site ou, le cas échéant, des conditions générales de la licence souscrite par votre établissement. Toute autre reproduction ou représentation, en tout ou partie, sous quelque forme et de quelque manière que ce soit, est interdite sauf accord préalable et écrit de l'éditeur, en dehors des cas prévus par la législation en vigueur en France. II est précisé que son stockage dans une base de données est également interdit. 


\section{ORGANISATION ET ACCOUNTABILITY DES ÉCHANGES LANGAGIERS LORS D'AUDITIONS JUDICIAIRES}

Esther GONZÁLEZ MARTÍNEZ 
$\mathrm{D}$ evant un ensemble donné de conversations, nous nous sommes demandé ce qui nous les faisait paraitre similaires. Comment expliquer que chaque échange du corpus donne l'impression d'être un exemplaire d'un même type ? D'où provient cet air de famille ? Pour percevoir cette ressemblance, point n'est besoin de savoir que ces conversations - étudiées uniquement à partir d'enregistrements sonores sont des auditions judiciaires, qu'elles font partie de procédures pénales françaises de comparution immédiate, qu'elles se déroulent toutes dans un bureau de la huitième section du parquet du tribunal de Paris, ni qu'elles réunissent un substitut du procureur de la République et une personne arrêtée pour des faits de nature correctionnelle ${ }^{1}$.

La similarité des échanges se rapporte en fait à un ensemble très important de traits : le nombre d'interlocuteurs, ce qu'ils font ensemble, leurs façons de parler, les topics qu'ils traitent, le déroulement et la durée de leur conversation... Il s'agit de phénomènes observables dans (et grâce à) une organisation particulière des échanges langagiers. Récurrents, ils sont produits in situ, en se parlant, par les interlocuteurs qui font ainsi émerger la conversation avec ses caractéristiques spécifiques.

\footnotetext{
* Cet article a été rédigé dans le cadre d'une recherche postdoctorale financée par le Fonds national suisse de la recherche scientifique (Subsides PA001-105069 et 81LA-68394). Nous remercions Jocelyne Berclaz, George Psathas, Philippe Sormani et Olivier Voirol de leurs remarques.

1. La procédure pénale française de comparution immédiate est activée à l'issue de l'enquête, lorsque le parquet considère que la simplicité de l'affaire et le caractère avéré des faits ne rendent pas nécessaire une instruction préparatoire. Dans ce cadre, après son arrestation par la police, un déféré, souvent arrêté en flagrance, est conduit devant le procureur qui, d'accord avec la loi, vérifie son identité, lui communique les charges retenues contre lui et prend ses déclarations si celui-ci en fait la demande. Suite à l'audition, le procureur fait comparaître le prévenu devant le tribunal correctionnel.
} 
La perceptibilité visuelle d'un phénomène, son caractère public (par opposition à privé), sa notoriété ou son degré de reconnaissance sociale - des caractéristiques qui définissent habituellement sa «visibilité » - requièrent que le phénomène soit en premier lieu «visible» au sens d'« intelligible $»^{2}$. Disponible aux acteurs, il fait partie du monde social où il apparait avec l'aspect qui le distingue; une propriété que l'ethnométhodologie saisit avec le concept central d'accountability ${ }^{3}$. Cette acception du terme ancre l'enquête empirique dont nous présentons ici les résultats dans la thématique de la visibilité. L'article décrit l'organisation d'un corpus d'échanges langagiers qui en fait des objets intelligibles, caractérisés par ailleurs par leurs contours équivoques ${ }^{4}$.

\section{UNE PROBLÉMATIQUE ETHNOMÉTHODOLOGIQUE}

«Les recherches en ethnométhodologie analysent les activités de tous les jours en tant que méthodes des membres pour rendre ces mêmes activités visiblement-rationnelles-et-rapportables-à-toutes-fins-pratiques, c'est-à-dire "accountable", en tant qu'organisation des activités ordinaires de tous les jours. $»^{5}$ Garfinkel note que la réalité sociale prend la forme de «choses organisationnelles »- activities, scenes of everyday life, familiar events, settings - qui se manifestent de façon similaire indépendamment des acteurs appelés à les accomplir ${ }^{6}$. L'accountability est la propriété de ces objets d'ordre d'être intelligibles, observables ${ }^{7}$, descriptibles, analysables,

2. Sur la perception pratique de la signification : QUERE, 1999.

3. Suivant l'usage, nous ne traduisons pas le terme accountability dont notre texte présente en détail la signification.

4. Notre corpus a été constitué à partir de l'ensemble des enregistrements audiovisuels (rushes) effectués par Raymond Depardon au Palais de justice de Paris pour son documentaire Délits flagrants (1994). Il recueille soixante-sept auditions judiciaires débouchant sur des procédures de comparution immédiate conduites par trois procureurs différents. Les auditions, réalisées dans une petite pièce en présence d'un gendarme, durent en moyenne sept minutes. En insigne représentant du cinéma direct, Raymond Depardon met en place des dispositifs d'enregistrement lui permettant de contrôler son influence sur la situation en question. Travaillant essentiellement sur les bandes-son, nous n'avons pas trouvé des éléments qui justifieraient de considérer le documentariste comme une partie de la conversation.

5. GARFINKEL, 1984 [1967], p. VII [trad. de l'auteur].

6. GARFINKEL, 1984 [1967], 2002.

7. La littérature ethnométhodologique utilise indistinctement les termes «observable» et «visible» (BACCUS, 1986, en est une exception), privilégiant néanmoins le deuxième lorsque la dimension visuelle du phénomène est importante. 
explicables, reproductibles, reconnaissables, typiques... Elle est l'œuvre des acteurs qui se rendent réciproquement disponibles les objets qui peuplent le monde social en même temps qu'ils le produisent. En se coordonnant autour d'une figure d'ordre, les acteurs donnent forme en commun à son accountability. Sur la base d'un accès partagé à l'objet qu'ils produisent, ils ajustent leurs comportements et poursuivent leur cours d'action.

Garfinkel insiste sur l'identité des méthodes de production et de l'objet et de son accountability; produire le phénomène signifie le rendre accountable ${ }^{8}$. Par ailleurs, le phénomène lui-même n'étant rien d'autre que le déploiement des méthodes qui le constituent, Garfinkel peut attribuer les mêmes caractéristiques au travail de constitution et à l'ordre qui en résulte : pratique, continu, il se fait sur place, suivant une logique endogène; il est entièrement l'œuvre des acteurs qui le produisent inévitablement, se référant nécessairement les uns aux autres; il s'accomplit à toutes fins pratiques, de façon reproductible et récurrente, "seen but unnoticed", comme la chose la plus ordinaire du monde.

«Voici ce qui retient mon attention quand j'utilise le terme accountable: j'entends observable-et-rapportable, c'est-à-dire à la disposition des membres à travers ces pratiques situées que sont voir-et-dire. ${ }^{9}$ Le sens de la paire observable-et-rapportable, récurrente dans les définitions fondatrices du concept d'accountability, est élaboré empiriquement par des travaux ethnométhodologiques d'activités axées sur la perception visuelle ${ }^{10}$. Des acteurs créent des champs phénoménaux dans lesquels ils repèrent et identifient des entités. Des archéologues, par exemple, rendent observables, en comparant leur couleur ou en traçant leur forme, des taches dans le terrain ${ }^{11}$. Le travail de mise en évidence du phénomène (cette chose-ci) va de

8. «[L]es activités par lesquelles les membres produisent et gèrent des situations de la vie quotidienne [settings of organized everyday affairs] sont identiques aux procédures des membres pour rendre ces situations "account-able". » (GARFINKEL, 1984 [1967], p. 1 [trad. de l'auteur]).

9. GARFINKEL, 1984 [1967], p. 1 [trad. de l'auteur].

10. CARLIN, 2003 ; GOODWIN, 1994 ; GOODWIN et GOODWIN, 1996 ; HEATH et LUFF, 1996 ; LYNCH, 1985 ; POLLNER, 1979 ; SHARROCK et WATSON, 1990. Sur le caractère accountable des déplacements dans des espaces publics : HESTER et FRANCIS, 2003 ; LEE et WATSON, 1992-1993; QUERE et BREZGER, 1992-1993; RELIEU et QUERE, 1998 ; RYAVE et SCHENKEIN, 1974 ; SUDNOW, 1972. Noter aussi les textes fondateurs de SACKS, 1972, 1984, 1989a, 1989b.

11. GOODWIN, 1994. 
pair avec sa désignation (une tache de couleur); les traits d'union (observable-et-rapportable) pointant bien vers ce rapport d'élaboration réciproque. La production de l'observabilité - mettre en avant, rendre saillant - et de la dicibilité - identifier, déterminer de quoi il s'agit apparaissent comme les deux faces indissociables d'un même geste pratique qui rend disponible un objet de connaissance.

L'analyse ethnométhodologique de conversations nous indique la signification de « voir-et-dire » lors de cours d'action dont la dimension visuelle n'est pas centrale. Elle observe des interlocuteurs produisant conjointement au long de la conversation des actions qu'ils rendent disponibles comme étant de tel ou tel type $^{12}$. La formulation du tour, son placement au sein de la conversation ou d'une activité particulière, et/ou l'identité du locuteur, aident à identifier l'action que A accomplit. Le récit qu'une épouse fait d'une journée difficile au bureau peut être une demande de sympathie, une sollicitation d'aide, une invitation à l'analyse ou la première partie d'un échange de nouvelles. Lorsque le récit, placé au début de la conversation, se conclut avec « et puis toi, tu as passé une bonne journée ? », c'est cette dernière action qui occupe le premier plan. La mise en évidence et l'identification de l'action que les interlocuteurs sont en train de produire est aussi l'œuvre du tour de B. Lorsque le mari répond en racontant sa journée, il exhibe qu'une action a eu lieu et l'identifie comme étant la première partie d'un échange de nouvelles. Encore une fois, rendre observable une action et déterminer de quoi il s'agit apparaissent comme des pratiques indissociables; identiques par ailleurs à la production même de l'action.

Dans ses écrits, Garfinkel fait référence à deux types d'accountability difficiles à distinguer parce que interdépendantes et pourtant incommensurables ${ }^{13}$. La production locale de détails phénoménaux et l'ordre qui en résulte auraient, en situation, une accountability «naturelle» qui émerge de l'intérieur même du cours d'action. L'accountability «classique » correspond aux descriptions, ordinaires ou scientifiques, qui reprennent le phénomène mais ne saisissent pas la cohérence contextuelle de sa production in vivo; elles participent à la production locale mais ce n'est pas par leur intermédiaire que le phénomène se constitue en premier lieu.

12. SCHEGLOFF et SACKS, 1973 ; SCHEGLOFF, 1990 [1968].

13. La réflexion sur les foyers de constitution de l'accountability est particulièrement technique (GARFINKEL, LYNCH et LIVINGSTON, 1981; GARFINKEL et WIEDER, 1992 ; GARFINKEL, 2002). Voir aussi : QUERE, 1985. 


\section{UNE ENQUÊTE EMPIRIQUE}

L'étude de l'organisation des échanges de notre corpus - décrits régulièrement comme des auditions de comparution immédiate - ne se situe pas au niveau de leur accountability classique. Nous ne pouvons certainement pas faire abstraction des descriptions disponibles de ces échanges: il s'agit d' "auditions" réunissant un "procureur» et un "déféré ». Cela ne nous oblige pas pour autant à faire de l'une ou l'autre de ces descriptions le point de départ (comment l'audition est-elle organisée ?) de notre enquête ou son point d'arrivée (qu'est-ce qui fait qu'il s'agisse d'une audition?).

Partir de la production locale d'ordre signifie s'exposer à «la masse écrasante des détails désespérément circonstanciels ${ }^{14}$ des échanges. Notre enquête se concentre moins sur la production de ces détails en soi que sur leur articulation dans des formes reconnaissables. Dès le départ, des détails sont rendus observables-et-rapportables; en s'alignant, ils font émerger des phénomènes d'ordre d'une autre dimension: des questions-réponses s'organisent en séries qui façonnent un échange de tel ou tel type. Nous donnant justement l'échange par unité d'étude, nous cherchons à présenter dans leur articulation des figures d'ordre récurrentes qui expliqueraient l'air de famille caractéristique de notre corpus.

Mais à quelle chose organisationnelle la conversation donne-t-elle forme ? Quel est le phénomène d'ordre que les interlocuteurs rendent observable-etrapportable in situ? Sacks affirme qu'une des difficultés de l'analyse de longues séquences conversationnelles est d'avoir affaire à des «choses » (things) dont nous ignorons l'existence ${ }^{15}$. Sa remarque nous met en garde contre une catégorisation préalable de la rencontre et de son accountability naturelle qui guiderait l'analyse. L'existence de cette chose-là, ce dont il s'agit et ses méthodes de production en tant que phénomène accountable font partie des découvertes de l'enquête.

En quelques pages, notre texte présente les principales caractéristiques de l'organisation d'un corpus étendu d'échanges ${ }^{16}$. La description détaillée de

14. GARFINKEL, 2001 [1996], p. 35.

15. SACKS, 1992, II, p. 354-355.

16. Pour une description détaillée : GONZÁLEZ MARTÍNEZ, 2003. 
leur production doit forcément céder la place à leur présentation et leur articulation dans un tableau général. Toutefois, pour exemplifier ces caractéristiques et fournir à nos lecteurs un lieu d'observation de leur émergence, nous ferons de multiples références à l'audition 26, reproduite intégralement en annexe.

\section{L'ORGANISATION DES ÉCHANGES}

Le procureur et le déféré commencent à se parler sans présentation préalable de leur rencontre, de son déroulement ou de ses objectifs. Après l'identification du déféré, le procureur introduit une discussion sur «ce qui s'est passé qui a donné lieu au défèrement » que son interlocuteur poursuit lorsqu'il prend la parole. L'introduction de cette «discussion » opère une segmentation dans l'échange et correspond au début de sa phase centrale sur laquelle nous avons concentré notre recherche. Cette discussion se termine lorsque les interlocuteurs enchaînent avec la poursuite de l'audition et initient les gestes qui conduisent à leur séparation physique. (Dans l'audition 26, la discussion se développe entre les lignes 2 et 171).

Le procureur et le déféré accomplissent la rencontre à travers une discussion sur «ce qui s'est passé ». S'ils s'accusent, se corrigent, se justifient, se mettent sous pression, se donnent tort ou raison, se présentent ou se ridiculisent, ils le font en discutant des faits reprochés. Au fil de la conversation, en parallèle à la production des différents éléments des faits, les interlocuteurs élaborent leurs perspectives sur ces éléments et sur l'organisation même de l'échange.

Les interlocuteurs conduisent la rencontre de façon fluide, les interventions s'enchaînant et se rapportant les unes aux autres. Le procureur et le déféré interagissent à travers des activités langagières, des formes d'échange avec une double dimension conversationnelle et pratique, qui font partie de leur bagage quotidien.

\section{Pluralité d'activités}

Les interlocuteurs commencent la discussion en s'exprimant chacun à leur tour sur «ce qui s'est passé ». A l'activité d'exhibition de perspectives 
correspond une séquence de deux interventions contiguës, composées régulièrement de longs tours à plusieurs unités ${ }^{17}$. Le procureur parle en premier suivi plus tard par le déféré; chacun s'exprime à son tour alors que son interlocuteur garde le silence ou produit des continuers (« oui », « mmh $\mathrm{mmh} »)$, des marques d'accord ou des répétitions. Dans l'audition 26, le procureur introduit un long tour (2-10), pendant lequel la déférée reste silencieuse, qu'il conclut en invitant son interlocutrice à prendre la parole (810) ; la déférée s'exprime dans les lignes 11-24 alors que le procureur se limite à des micro-interventions qui appuient le développement des déclarations. Le procureur introduit la discussion sur «ce qui s'est passé » sous l'angle des faits reprochés (un vol de portefeuille et un vol de portefeuille ou de porte-monnaie dans le cas de la déférée D. 26). Ainsi présentés, les faits ont un personnage central, censé correspondre au déféré, auteur d'une infraction. Le tour du procureur fonctionne comme une préface qui invite des déclarations, les cadre et leur fournit des matériaux pour se développer. Comme dans l'audition 26 («bien alors je vous écoute vous avez quelque chose à me dire ?»(8-9)), le transfert de la parole passe régulièrement par une invitation à faire des déclarations plus importantes qu'une simple reconnaissance des faits. En prenant la parole, le déféré se définit par rapport à l'intervention du procureur à partir de ses propres perspectives. Il inscrira régulièrement l'infraction dans un cours d'action ou une situation qui ré-élabore «ce qui s'est passé » et qui éventuellement le disculpe ; c'est ainsi, par exemple, que la situation de la déférée D. 26 (Noël, accueil de sa fille, manque de moyens, cohabitation avec ses parents, envie d'offrir des cadeaux...) prend le devant des faits reprochés qu'elle sert à expliquer.

A d'autres moments de la discussion, souvent à la suite des déclarations du déféré, les interlocuteurs entrent dans un échange questions-réponses au cours duquel ils développent une description unique des faits (25-58) ${ }^{18}$. Lors de cette activité - l'alignement de perspectives - le procureur produit une série de questions sans problématiser les réponses qu'il reçoit. Dans l'audition 26, cette activité s'initie avec l'intervention «quelle est votre situation ? ben oui » (25) suivie de la réponse de la déférée. Lors des paires

17. Le fonctionnement de cette activité correspond à l'enchaînement de stories (second stories, series of stories, invited stories) à l'intérieur d'une conversation (CUFF et FRANCIS, 1978 ; JEFFERSON, 1978 ; RYAVE, 1978 ; SACKS, 1974, 1978, 1992).

18. Sur les enchaînements question-réponse: CONEIN, 1987 ; HERITAGE et ROTH, 1995 ; MCHOUL, 1987 ; MERRIT, 1976 ; SACKS, 1987 et 1992. 
question-réponse suivantes, le procureur manifeste un accueil neutre ou positif des réponses de la déférée en s'abstenant de soulever des contrastes sur les faits. La déférée dit que c'est la situation avec ses parents qui l'a fait « craquer » et le procureur enchaîne sur cet élément pour l'interroger sur sa consommation de drogue. Les éléments des faits ainsi élaborés viennent enrichir le portrait de la situation de la déférée sans qu'ils entrent en contradiction les uns avec les autres. Certaines questions («vous êtes sortie de prison il y a longtemps? » (30), «il vous en faut combien une euh de l'héroïne ? (52)) reçoivent des réponses qui paraissent imprécises, mais le procureur ne s'appesantit pas pour autant.

L'échange devient ouvertement polémique lorsque les interlocuteurs alignent des contre-assertions - des énoncés avec la forme « $\mathrm{X}$ mais $\mathrm{Y}$ »-dans une séquence de dispute ${ }^{19}$. Pendant les séquences d'opposition de perspectives, les interlocuteurs continuent à élaborer «ce qui s'est passé », mais produisent des contrastes entre les éléments en circulation, rendant difficile leur intégration dans une même description du réel. Les interlocuteurs ne se servent pas de leurs tours pour concilier des propositions incompatibles sur les faits, mais les réaffirment, faisant ainsi émerger des positions contraires. Dans l'audition 26, la dispute se développe (imbriquée à un échange questions-réponses) entre les lignes 133 et 168 ; le procureur peine à croire que l'amie de la déférée n'était pas au courant des vols, comme celle-ci l'affirme. Les disputes se perpétuent en se complexifiant, les éléments en circulation augmentent, les interlocuteurs les contestent immédiatement, les délaissent, les reformulent, reprennent un élément présenté au début de la dispute pour le réaffirmer ou le remettre en question. Dans l'audition 26 encore, le procureur fait référence aux observations de la police (138), au fait que les deux amies se sont connues en prison (150) et qu'elles connaissaient probablement toutes les deux le vol à la tire (151). Les points en discussion varient, mais restent liés à un même aspect des faits (la participation de l'amie au vol) qui se définit en même temps que la position des interlocuteurs à son propos.

19. Les séquences de dispute ont été étudiées par COULTER, 1990; GOODWIN, GOODWIN, 1997 ; GRUBER, 1998 ; HUTCHBY, 1996 ; LYNCH, 1982 ; MAYNARD, 1985. 
$\mathrm{Au}$ cours de chaque rencontre, le procureur rédige la «déclaration » du déféré qu'il note sur le procès-verbal d'audition ${ }^{20}$. Comme dans l'audition 26, la séquence de rédaction (62-129) est régulièrement imbriquée à des échanges question-réponse ou à des disputes. Lors de l'activité d'attribution de perspectives, le procureur formule et oralise les différentes unités de la déclaration en vue de leur inscription («je reconnais les faits» (62-63)). Il propose, plus ou moins clairement, ces éléments au déféré qui les accepte, les reformule ou les complète. Le procureur produit aussi régulièrement des formulations qui décrivent, résument ou expliquent ce qui est fait et ce qui est dit, ainsi que la façon de le faire («donc je marque » (62)) ${ }^{21}$. C'est ainsi que le procureur, secondé par son interlocuteur, attribue à celui-ci et des déclarations sur les faits et l'initiative d'un échange type «vous dites, je note » où le magistrat n'agirait qu'en simple inscripteur.

\section{Alternance et enchevêtrement d'activités}

Les interlocuteurs changent d'activité en produisant des enchaînements différents de tours: multi-unit turns, questions-réponses, séries de contreassertions ou d'oralisations d'inscription. Selon le mode d'échange, les interlocuteurs, essentiellement, s'écoutent parler, s'accordent ou s'opposent sur «ce qui s'est passé », ou bien le fixent par écrit. Le changement d'activité fonctionne également à un autre niveau; il permet de reprendre, sur des modes divers, les éléments des faits abordés auparavant. Le procureur peut dire que le déféré a volé, le laisser soutenir après qu'il n'est pas l'auteur d'un vol, lui poser ensuite des questions qui permettent de développer les perspectives du déféré (où se trouvait-il au moment des faits ? a-t-il assisté au vol ? connaissait-il son auteur ?). Il contestera plus tard la description des faits engendrée par la discussion précédente, pour finir par prendre note quand même de la «déclaration » du déféré.

Outre qu'elles s'alternent, les activités sont enchevêtrées les unes aux autres ${ }^{22}$; les interlocuteurs balancent alors très rapidement entre exposer des perspectives, s'y aligner, s'y opposer ou les fixer par écrit. Parfois, les

20. Sur la production d'un texte au cours d'une interaction : CICOUREL, 1975 ; KOMTER, 2001 ; PSATHAS, 1990 ; ZIMMERMAN, 1992b.

21. Sur les formulations: GARFINKEL, SACKS, 1986 [1969].

22. JEFFERSON et LEE, 1992 [1981]; SCHEGLOFF, 1988-1989; WHALEN, ZIMMERMAN et WHALEN, 1992a, analysent des conversations comprenant changement et enchevêtrement d'activités. 
interlocuteurs introduisent, au sein d'une séquence réglée sur un format, des interventions ou des séquences propres à un autre type. A d'autres moments, la discussion s'alimente de plusieurs formes d'échange se développant en étroite symbiose. Le percement et l'imbrication d'activités parcourent l'échange en l'organisant : dans le tour de la communication des charges, le procureur crée de petites ouvertures (des «ajours») à travers lesquelles il laisse percer la version du déféré tout en contrôlant strictement sa parole ${ }^{23}$; le déféré désarticule la présentation des faits reprochés en contestant ce qui est dit; avant de poursuivre la communication des charges, le procureur pose au déféré quelques questions pour s'entendre sur un point précis; le procureur sollicite les déclarations du déféré, mais il les guide fortement par des interventions autres que de simples continuers ou des répétitions; le déféré est en train de produire ses déclarations, le procureur l'interrompt avec des questions, le déféré répond et reprend immédiatement son discours; le procureur et le déféré élaborent « ce qui s'est passé » à travers un échange questions-réponses, mais les interventions du procureur problématisent plus ou moins subtilement la description des faits; ailleurs, le procureur combine habilement des oralisations de la déclaration écrite avec des questions et des contre-assertions qui développent ou contestent la description de «ce qui s'est passé » mise en avant par (ou attribuée à) son interlocuteur.

Dans l'audition 26, après une séquence typique de communication des charges+déclarations (2-24) suivie d'une série questions-réponses (25-58), le procureur introduit la rédaction de la déclaration (62). Les interlocuteurs restent clairement dans cette activité jusqu'à la ligne 129 , où le procureur annonce la suite de la rencontre. Dans cette longue séquence, les interlocuteurs passent très rapidement de la rédaction de la déclaration au développement de certains aspects des faits ou à une opposition à leur propos, pour finalement revenir toujours à la rédaction. Ils le font en alternant et imbriquant oralisations d'inscription, questions-réponses et contre-assertions. A plusieurs reprises, les interlocuteurs formulent un élément de la déclaration qui est complété uniquement après un échange interrogatif. En 88, le procureur initie un tour au départ interrogatif («pourquoi ? ) qui prend finalement une forte tonalité polémique («c'était pas le moment de craquer » (93)). Ailleurs, des expressions d'accord («bon je marque » (110), «ça s'est sûr» (127)) déguisent à peine le mécontentement du procureur vis-à-vis des réponses obtenues. Plus tard

23. GONZÁLEZ MARTÍNEZ, 2001. 
dans la discussion, lorsqu'ils traitent de la participation de l'amie de la déférée au vol (133-168), les interlocuteurs imbriquent un échange questions-réponses à une dispute. Dans un échange interrogatif marqué par des questions répétées ou rhétoriques, et l'accumulation de «pourquoi ? », il s'agit plus de mettre en doute et de défendre la version de la déférée que de la développer.

Même si certaines positions paraissent relativement stables, les différentes activités se suivent et se recouvrent sans respecter d'ordre ou de hiérarchie fixe. Les interlocuteurs établissent en situation des arrangements d'activités très divers. Dans l'audition 26, par exemple, le procureur annonce la suite de la rencontre (129) - un acte qui débute régulièrement la clôture de l'audition - pour, tout de suite après, continuer à examiner les faits et même compléter la déclaration de la déférée. Bien que les interlocuteurs soient toujours brefs, le temps qu'ils passent dans les différentes activités varie aussi d'une discussion à une autre : des présentations des faits reprochés très détaillées prennent dans certaines discussions la forme d'une simple énumération des charges; parfois quelques questions suffisent; il n'y a pas forcément de dispute et l'on ne s'appesantit pas sur les points en conflit; soit la déclaration écrite est montée d'un trait soit la rédaction s'étend sur plusieurs séquences. Le maintien d'une activité ou son abandon ne sont pas déterminés par les éléments des faits en élaboration (tel élément suscite beaucoup d'attention lors de telle discussion et non pas lors de telle autre, ou bien suscite des échanges très divers). L'alternance et l'enchevêtrement d'activités ne sont pourtant pas des phénomènes erratiques.

\section{Etablissement d'un « je » multiple et rassembleur}

Les changements d'activité alimentent un mouvement de configuration d'une importance particulière : l'établissement d'un «je »- le protagoniste de la déclaration écrite, censé représenter le déféré - multiple et rassembleur. Ce mouvement opère un basculement du « vous » propre aux interventions du procureur sur le «je» des propos du déféré; celui-ci s'exprimant progressivement à travers les attributions que le procureur lui confère jusqu'à parvenir à la signature de la déclaration.

En tant que figure, le « je » de la déclaration écrite est multiple. Il s'agit d'un je déclaratif - qui manifeste son désir de faire des déclarations -, affirmatif qui avance des propositions qu'il soutient comme vraies -, inculpé - qui se 
prononce par rapport à une accusation -, acteur - sujet des actes décrits dans la déclaration - et (peut-être) responsable, doté d'intention et pourvu de motifs. Le «je » est aussi rassembleur parce qu'il dit, ne serait-ce qu'à la forme négative, les différentes perspectives sur les faits exprimées au long de la discussion, y compris celles du procureur, des policiers ou des victimes, que le déféré aurait de la peine à endosser.

La déférée D. 26 commence ses déclarations en enchaînant sur la description de ses actes que le procureur vient de faire («oui j'ai fait ça parce que » (11)). Ensuite, lors de la séquence interrogative (25-58), elle se prononce par rapport à sa sortie de prison et à sa consommation de drogue; des éléments introduits dans la conversation (tout du moins explicitement) par son interlocuteur. Le procureur pose des questions qui se transforment en affirmations lors des réponses de la déférée et qui paraissent comme la poursuite de ses déclarations. En initiant la rédaction, le procureur attribue à la déférée la déclaration «je reconnais les faits j'ai commis ces vols » (6263). La déférée continue à parler de sujets que le procureur introduit dans la discussion (situation et âge de sa fille $(69,77)$ ) et accepte l'interprétation que le procureur fait de ses déclarations initiales (117-129) et de ses réponses (voir l'élaboration de "je n'avais pas volé depuis ma sortie de prison en juillet »). Les propos de la déférée sont repris dans la déclaration écrite, mais c'est le procureur qui sélectionne dans son discours ce qu'il convient de noter, le formule et décide de l'ordre dans lequel doivent apparaître les différents éléments (la phrase sur la consommation d'héroïne est ainsi placée in fine (169-170)). Ce travail de ventriloquie complexe, où chaque interlocuteur est le véhicule des perspectives de l'autre, mais où certaines perspectives priment, passe par la production des différentes activités. En enchaînant déclarations, questions-réponses, dispute ou rédaction, les interlocuteurs développent et reprennent, sur différents modes, les éléments des faits, tels les motifs de la déférée, sa sortie de prison et les vols commis éventuellement depuis. Ces éléments se modulent en se déplaçant entre les discours des deux interlocuteurs pour finir par apparaître dans la déclaration.

Mises en série, les différentes oralisations donnent la déclaration suivante : « je reconnais les faits j'ai commis ces vols car je devais recevoir ma fille de sept ans au domicile de mes parents où j'habite elle vit actuellement (placée chez une nourrice) je voulais de l'argent pour lui offrir des cadeaux de Noël en ce moment je craque car nous sommes huit à vivre chez mes parents et je suis à leur charge je n'avais pas volé depuis ma sortie de prison en juillet j'ai 
repris la consommation d'héroïne depuis une semaine». Cette déclaration s'organise autour d'un «je»: déclaratif, affirmatif, inculpé, acteur, responsable, doté d'intention et pourvu de motifs. Parmi ces motifs, on trouve ceux qui sont (et ont été) invoqués explicitement - avoir de l'argent pour offrir des cadeaux de Noël - et celui qui est suggéré en pointillés par le simple alignement des affirmations. Un contraste pourrait être établi à partir des éléments inscrits : la déférée se dit sans ressources et consommatrice d'héroïne. Le désaccord qui a traversé toute la rencontre, sans s'exprimer ouvertement, se retrouve dans la déclaration sous forme d'énigme «comment la déférée paye-t-elle sa consommation si elle n'a pas d'argent?».

\section{Maîtrise asymétrique de l'échange}

L'audition est accomplie par les deux interlocuteurs. Toutefois, ce sont les initiatives du procureur qui orientent le plus clairement l'élaboration des faits et l'organisation de l'échange ${ }^{24}$. Le procureur mobilise un répertoire plus large de types d'énoncés : invitations à faire des déclarations, questions, contre-assertions, oralisations d'inscription. En exploitant ces ressources, le procureur met en marche des dynamiques organisatrices dans lesquelles le déféré est régulièrement entrainé. Modifier le mode d'échange donne au magistrat une maitrise considérable sur la formulation et la réception de ses interventions et de celles du déféré; il peut sortir d'une dispute en passant à la rédaction, le déféré abandonne ses déclarations parce que le procureur lui pose une question, la discussion est finie lorsque le procureur présente la suite de l'audition.

Le procureur décide de l'activité, de son emplacement et de sa durée; dans la discussion 26 par exemple, c'est le procureur qui lance et abandonne en premier la communication des charges, les questions-réponses, la rédaction. Le procureur initie les activités et ses interventions ouvrent ensuite les paires des tours qui les composent. Lors de la présentation des charges, le procureur introduit la discussion sur « ce qui s'est passé » qu'il oriente tout au long de la rencontre. Le magistrat invite le déféré à se manifester («bien alors je vous écoute vous avez quelque chose à me dire?»(8-9)), l'encourage à poursuivre ses déclarations ou les relance. Dans l'audition 26

24. Sur l'asymétrie des ressources lors d'interactions formelles: EGLIN et WIDEMAN, 1986 ; HAVE, 1991 ; MAYNARD, 1991. 
encore, lignes $13,15,19,23$, le procureur se limite à des énoncés échos; dans d'autres auditions, il articule: «et ensuite», «reprenez vos déclarations », «continuez ». Les questions sont l'œuvre du procureur qui signale le type de réponse recherchée. Parce que les tours du déféré constituent la deuxième partie des paires qui donnent forme à la discussion, seul le procureur initie vraiment les disputes. Les séquences de synthèse, de transition et les pré-séquences répondent aussi à l'initiative du magistrat; dans l'audition 26 , le procureur introduit une pré-séquence de type résumé qui fait la transition entre la séquence interrogative et la rédaction (58-61). Le procureur est libre d'écrire, de proposer ou d'oraliser les éléments de la déclaration écrite qu'il façonne et ordonne; alors que la consommation de drogue est abordée presque au début de la discussion 26, le procureur décide d'en faire référence au niveau de la déclaration écrite seulement vers la fin. Le procureur manie avec adresse les formulations qui décrivent l'échange et son ordre («bien donc pour revenir aux faits » (58-59), « je marque » (62), «bien alors je vais simplement compléter » (168)). Il ne se sent pas obligé de respecter la continuité - au niveau thématique et du type d'énoncé - entre ses interventions et celles de son interlocuteur. Il n'est même pas sûr qu'il réagisse aux remarques du déféré. Enfin, seul le procureur se permet de clore la discussion et de mettre fin à la rencontre.

Les interventions du déféré viennent toujours en deuxième lieu par rapport à celles du procureur qui les oriente clairement. Parfois, le déféré élabore les faits en s'éloignant des pertinences de son interlocuteur; réclame la parole; rechigne à commencer, à poursuivre ou à abandonner ses déclarations; reprend ses déclarations après que le procureur les ait interrompues; suspend ou met fin à l'intervention de son interlocuteur en produisant des énoncés qui deviennent, en situation, véritablement disruptifs ou conclusifs; tient un discours parallèle avec le procureur, des énoncés des deux parties s'intercalant alors rapidement sans rapport apparent. La déférée D. 26, par exemple, fait preuve d'un contrôle remarquable de son discours : elle limite ses déclarations ( «c'est tout » $(20,21-22)$ ), se permet des réponses évasives (30-37, 52-58, 117-127), coupe court à la dispute sur la complicité de son amie et persévère dans ses déclarations initiales (133-168). Il n'en reste pas moins que les interventions du procureur constituent des propositions par rapport auxquelles le déféré définit son discours. Le déféré reformule ces propositions, les rejette ou les accepte. Néanmoins, ses interventions sont saisies à l'intérieur d'un mode d'organisation de l'échange qui donne au procureur le rôle directeur. 


\section{UNE ACCOUNTABILITY TRANSPARENTE, MOUVANTE ET ÉQUIVOQUE}

Pour les interlocuteurs, l'accountability de l'échange - son caractère observable-et-rapportable - est une affaire pratique réglée in situ. $\mathrm{Ni}$ observateurs détachés ni analystes a posteriori, les interlocuteurs produisent constamment les interventions qui donnent forme à l'échange. Ils ne peuvent pas (au sens d'exigé et d'inéluctable) entretenir la conversation sans (se) la rendre à tout moment intelligible. Sur le plan de l'organisation pratique, les interlocuteurs ne font rien d'autre que d'ajouter de nouvelles interventions qui rendent disponible «ce dont il s'agit ». Inséré dans une conversation, un énoncé est identifiable comme accomplissant telle ou telle action. Il qualifie également les énoncés qui, de façon immédiate ou non, le précèdent et le suivent; une réponse, par exemple, repère une question dans l'énoncé précédent et projette une nouvelle question comme une suite possible. Des énoncés accountable émergent ainsi progressivement en même temps que l'échange qu'ils composent (un échange interrogatif par exemple) avec sa propre accountability, le tout s'élaborant réciproquement. D'un côté, en s'enchaînant, les énoncés (et les actions qu'ils véhiculent) produisent et identifient l'échange auquel ils participent. D'un autre, la production et l'accountability des énoncés dépendent de l'échange dans lequel ils s'insèrent.

L'organisation et d'énoncés et d'un échange observables-et-rapportables n'est pas forcément un processus linéaire, unidirectionnel, qui enchaîne tous les gestes des interlocuteurs dans une seule ligne d'action et d'accountability. Les énoncés des acteurs sont susceptibles de véhiculer en situation plusieurs actions qui ne cessent de se multiplier au long de l'échange. Certaines sont coupées du reste, d'autres s'alignent dans des formes reconnaissables qui se déroulent (parfois simultanément) pendant une partie de la conversation ou bien d'un bout à l'autre. Certains enchaînements s'intègrent dans un mouvement plus large, d'autres écartèlent l'échange dans plusieurs directions. De ces interrelations complexes entre les énoncés et l'échange en formation dépendent la production et l'accountability de ce que les interlocuteurs accomplissent à chaque moment ainsi que sur l'ensemble de la conversation.

Comment cela se présente-t-il dans les échanges de notre corpus ? Au début de la discussion, les reproches ouvrent un espace pour tout un ensemble 
d'actions : accusations, excuses, justifications, aveux, dénis, explications, dénonciations... Néanmoins, tout aussi rapidement, la conversation est organisée comme une audition où il s'agirait d'écouter le déféré et de prendre note de ses déclarations. Ces deux lignes d'action sont canalisées dans une troisième - particulièrement saillante et traversant la discussion d'un bout à l'autre - qui les développe et concilie : déterminer « ce qui s'est passé ». Les interlocuteurs vont alors élaborer les faits dans une alternance et un enchevêtrement de modes d'échange qui leur permettent également de développer une audition et d'accomplir des actions à la suite des reproches. En introduisant des éléments des faits, en les développant, les rejetant ou les transformant, les interlocuteurs déterminent « ce qui s'est passé », répondent aux reproches (les éléments véhiculant des accusations, justifications, aveux) et produisent une audition où les déclarations du déféré sont entendues et recueillies par écrit. Le façonnement, tout au long de la rencontre, du «je » censé représenter le déféré cristallise cette organisation où différentes choses, souvent contradictoires, sont faites en même temps, les unes par l'intermédiaire (et parfois sous couvert) des autres.

La complexité des échanges n'est pas la conséquence de la faillite d'un mode d'organisation plus simple qui ne réussirait pas à s'imposer. Ce qui est à l'œuvre est un mode d'organisation en soi complexe, orchestré largement par le procureur, qui réussit particulièrement bien à ordonner chaque échange ${ }^{25}$. Façonné à partir des éléments exposés ci-dessus, ce mode d'organisation est à l'origine d'une articulation d'accountabilities qui, dans leur alternance et leur superposition, donnent forme à un échange dont l'accountability générale est mouvante et équivoque. Elle est mouvante parce que ce dont il s'agit (accuser, examiner les faits, produire des déclarations...) n'est ni unique ni stable. Le procureur reproche des infractions, écrit une déclaration qui disculpe le déféré, se met à l'écoute de ses déclarations, le fait se prononcer sur des éléments que celui-ci n'a pas abordés... L'accountability est équivoque parce que ce dont il s'agit peut se rapporter à différentes choses à la fois. On peut se demander ce que fait exactement le procureur lorsqu'il dit «je vous écoute» ou «les policiers vous reprochent» ou lorsqu'il «prend note» des déclarations. Pourtant, parce que la conversation est très brève, parce que ce que les interlocuteurs font à un certain niveau est extrêmement simple (détermination fort limitée de «ce qui s'est passé » à l'intérieur de modes d'échange ordinaires) et

25. Notre réflexion suit ici JEFFERSON, 1988. 
parce que le procureur formule, segmente et fait avancer en permanence la conversation, celle-ci est aussi, à un certain niveau, parfaitement transparente. Ce dernier fait, loin de contredire la thèse d'une accountability mouvante et équivoque, ne fait que la compléter l'accountability serait aussi transparente - et la justifier davantage; la conversation est d'autant plus complexe qu'elle peut paraitre par moments évidente. En labyrinthe de miroirs, la conversation produit, combine et exhibe différentes vues de «ce dont il s'agit». Percevoir cette complexité, trouver un mode de circulation pour s'y retrouver, la développer ou la limiter afin d'organiser l'échange dans un sens précis, sont autant d'épreuves où se mesurent les ressources des interlocuteurs.

Ce mode d'organisation rend possible la rencontre telle que nous la connaissons. Celle-ci n'aurait pas lieu si le procureur se limitait à accuser le déféré ou si, en arrivant, il disait «veuillez me dicter la déclaration à faire apparaître dans le procès-verbal ». L'échange se tisse parce que le déféré ne garde pas le silence, en reste à des aveux ou dit «voici la déclaration que je vous demande de noter ». Le procureur réintroduit les reproches en guidant la discussion sur les faits; parce qu'il s'appuie quelque part sur ce que son interlocuteur lui dit, il peut prétendre être en train de recueillir ses déclarations. La composante audition disparaîtrait complètement si le procureur refusait d'entendre la version du déféré ou la contestait d'un bout à l'autre.

Notre analyse fait écho pour finir aux descriptions sociologiques, juridiques ou journalistiques de l'audition - qu'elles plaident en sa faveur ou en sa défaveur -, aux comptes rendus des propres acteurs et des observateurs. Ces descriptions, en elles-mêmes et dans leur diversité, expriment à chaque fois que l'audition est une situation ambiguë (les erreurs d'appellation des protagonistes, les difficultés à lui donner une dénomination ou à en arrêter la nature juridique en sont une preuve $)^{26}$. Et cela notamment à cause de la

26. Les déférés qui penseraient que leur sort se décide au cours de l'audition, s'étonneraient d'apprendre que la couleur du dossier (rose = comparution immédiate), (vert = comparution par procès-verbal) indique la suite de la rencontre. Les déférés demandent «et le procureur, je vais le voir aujourd'hui ? » ou « vous me condamnez à quoi vous?». Les procureurs doivent préciser qu'ils ne sont ni des policiers, ni des éducateurs, ni des assistants sociaux. En partant, certains déférés remercient les procureurs ou veulent leur tendre la main, ceux-là leur font remarquer «qu'on n'est pas vraiment dans une position amicale» ou qu'ils ne savent pas s'il faut les remercier. Les procureurs conduisent des auditions, mais, à-côté de la porte d'entrée de la pièce où celles-ci ont lieu, un écriteau indique «Interrogatoire en cours. Ne pas déranger ». Dans certains comptes rendus de l'audition ou du documentaire Délits flagrants, 
complexité du rôle du procureur, accentuée dans le cadre de la procédure de comparution immédiate par l'absence d'instruction. Le procureur est placé à la tête de l'enquête tout en menant l'accusation et en incarnant l'action publique $^{27}$. Il se situe entre l'homme qui accuse et celui qui cherche une vue d'ensemble des faits, y compris parfois des éléments favorables au mis en cause. Alors qu'il est censé essentiellement entendre le déféré, il oriente décidément la teneur de ses propos. Malgré un cadre légal qui ne s'y prête pas, le procureur va au-delà de la simple notification ou du recueil de déclarations, sans pouvoir se permettre un véritable interrogatoire. A son tour, le déféré est mis dans une position de balancement entre la confrontation, la soumission, la résistance et la collaboration à une élaboration des faits qui pourrait éventuellement le disculper. Tout cela à un moment du processus pénal qui se doit expéditif, dont les contours sont à la fois élémentaires et vagues, où il serait encore possible de modifier la suite des événements, mais où les jeux sont en réalité largement faits.

\section{Conclusion}

Suggérer que l'accomplissement d'un cours d'action est identique à la production de son intelligibilité ouvre une perspective nouvelle pour l'étude des phénomènes sociaux. Celle-ci demande de mettre l'accent sur l'organisation des activités quotidiennes pour comprendre les processus par lesquels ces activités deviennent disponibles en prenant forme. Le mouvement central serait un faire procédural qui, en nourrissant le cours d'action, saisit ce dont il s'agit et projette une suite. Placées en dehors de la conscience, dans le domaine des pratiques, cette production et cette phénoménalisation des rencontres deviennent une affaire partagée, élaborée en commun par les acteurs en interaction. Avançant sur différents plans, ce faire qui fait sens, ne parviendrait pas à tisser des cours d'action complexes s'il n'enchaînait pas les éléments en circulation. Le chercheur se doit d'identifier les ordres particuliers qui se créent à l'intérieur des textures de détails phénoménaux qui sont les activités sociales. Les fils dans lesquels sont pris ces détails, certains plus évidents ou fondamentaux que d'autres,

le déféré est appelé le prévenu, l'inculpé ou le coupable, le procureur est pris pour un juge ou un juge d'instruction; la rencontre - que le code de procédure pénale ne qualifie pas - est qualifiée d'audition, d'interrogatoire, d'entretien ou de notification.

27. STEFANI, LEVASSEUR et BOULOC, 1993 [1964], examinent la nature juridique des actes de l'enquête de flagrance. 
traversant les rencontres en s'entrecroisant, leur donneraient leur identité et leur spécificité. Les acteurs se distinguent par leur participation à ces agencements, qui sont la trame et la matière même de leurs cours d'action. $\mathrm{Si}$, pour les produire, ils semblent suivre des structures familières, celles-ci sont autant les ressources qu'ils mobilisent que le fruit de leurs pratiques; elles servent à identifier et à produire un cours d'action qui leur est pourtant irréductible. 


\section{ANNEXES}

\section{CONVENTIONS DE TRANSCRIPTION ${ }^{28}$}

P. (suivi d'un numéro d'identification) procureur

D. (suivi d'un numéro d'identification) déféré

G.

gendarme

\begin{tabular}{|c|c|}
\hline$=$ & enchaînement \\
\hline[ & chevauchement \\
\hline - & silence (absence de parole) de moins d'une seconde \\
\hline$(1.03)$ & $\begin{array}{l}\text { silence (absence de parole), durée indiquée en minutes et } \\
\text { secondes }\end{array}$ \\
\hline : & allongement vocalique \\
\hline & intonation descendante \\
\hline & intonation progressive \\
\hline ? & intonation montante \\
\hline$?$, & $\begin{array}{l}\text { l'intonation montante devient progressive vers la fin du } \\
\text { segment }\end{array}$ \\
\hline ! & ton exclamatif \\
\hline$\uparrow \uparrow$ & $\begin{array}{l}\text { le segment encadré de flèches ascendantes est énoncé à un } \\
\text { niveau sonore plus élevé }\end{array}$ \\
\hline ors & les appuis sont soulignés \\
\hline & le segment encadré de degrés est énoncé très doucement \\
\hline$<$ & $\begin{array}{l}\text { le segment encadré de signes «moins que » est énoncé plus } \\
\text { rapidement }\end{array}$ \\
\hline
\end{tabular}

28. Développées à partir du système jeffersonien (ATKINSON et HERITAGE, 1984, p. IX-XVI). 
$\langle\quad$ le segment encadré de signes «plus que » est énoncé plus lentement

coup de glotte, claquement de la langue, sifflement

()

segment non transcrit parce que totalement incompréhensible, ou transcrit de façon incomplète ou peu sûre

(N...) segment non transcrit pour des raisons de confidentialité. $\mathrm{N}=$ nom $; \mathrm{P}=$ prénom $; \mathrm{j}=$ jour $; \mathrm{m}=$ mois $; \mathrm{a}=$ année ; $\mathrm{L}=$ lieu (ville, arrêt de métro, gare, quartier); L1 L2 = les deux lieux sont différents

(( il lit)) commentaire du transcripteur, rend compte des caractéristiques de l'énonciation ou identifie l'origine d'autres phénomènes sonores que la parole

fin du phénomène 


\section{AUDITION N ${ }^{\circ} 26$}

$1 \quad$ P.3 : alors vous êtes $\left(\right.$ N...P...) ${ }^{\circ}$ voilà ${ }^{\circ}$ vous êtes née le (j...m.......) (L...arr...) (0.02) 个et donc euh $\uparrow$ il vous est reproché comme vous avez reconnu euh devant la police deux vols >j'ai retenu deux vols $<$ le vol du: d'un portefeuille - au préjudice d'une dame eu:h ${ }^{\circ}(\mathrm{P} . . . \mathrm{N} . . .)^{\circ}$ et le vol d'un: portefeuille ou porte-monnaie au: préjudice d'une dame qu'on n'a pas pu identifier $\sqrt{ }$ ou d'un homme qu'on n'a pas pu identifier hein puisque c'est ce que vous avez dit à la police $\uparrow$ bien alors je vous écoute $\uparrow$ vous ave:z (quelque) chose à me dire ? donc euh,

D.26: oui j'ai fait ça parce que c'est les fêtes de Noël et j'ai pas ma fille à ma garde et eu:h je=

P.3: =vous n'avez pas votre fille $=$

D.26: $=$ à ma garde $=$

P.3: =à votre garde oui

D.26: e:t je ne l'ai que pendant les fêtes et j'ai pas d'argent j'ai rien donc je l'ai que quand elle vienne chez chez ses grands parents donc chez chez moi quoi=

P.3: =oui chez vos parents oui

D.26: (qu') elle ait au moins ses cadeaux c'est tout mais (ça fait de ça fait) très longtemps que ${ }^{\circ} \mathrm{j}$ 'avais pas volé ${ }^{\circ}(0.01)$ c'est tout=

P.3: =que vous n'aviez pas volé

D.26: j'ai demandé les aides financières on me refuse tout le temps

P.3: quelle est votre situation ? ben oui.=

D.26: =je suis sans profession

P.3: vous êtes $=$

D.26: =je (suis) chez mes parents je craque - on me demande de l'argent j'en ai pas

P.3: oui vous êtes sortie de prison il y a longtemps?

D.26: il y a longtemps et moi moi au mois de juillet depuis le mois de juillet je suis [tranquille

P.3: $[(\mathrm{mmh})$

D.26: je: je suis en mise à l'épreuve ça va ça se passe très bien - et voilà quoi. 
36 P.3: oui

37 D.26: j’ai craqué

P.3: ouais vous avez - dit au policier et d'ailleurs c'est confirmé par la découverte d'u:ne d'une seringue je crois vous êtes toujours toxicomane?

D.26: oui

P.3: euh vous prenez toujours de la drogue ?=

D.26: =oh là là depuis une semaine j'arrête pas entre mes parents qui me font craquer

P.3: vous n'arrêtez pas ?

D.26: depuis une semaine

P.3: [ouais

D.26: [je n'arrête pas=

P.3: =ouais $(0.01)$ ((est en train d'écrire $))$ d'accord et vous prenez quoi de l'héroïne ?

D.26: de [l'héroïne

P.3: [oui (0.01) et: il vous en faut combien une euh de l'héroïne ?,

D.26: pas beaucoup ça fait une semaine

P.3: - que vous n'en avez pas pris attendez [个j'ai pas (tout) compris $\uparrow$

D.26: [ $\uparrow$ non $\uparrow$ ça fait ça fait une semaine que j'ai que j'ai re que j'ai repris

P.3: que vous avez repris donc c'est ça (0.01) bien donc pour revenir aux faits euh donc vous reconnaissez vous contestez pa:s=

D.26: $={ }^{\circ}$ bien sûr ${ }^{\circ}$

P.3: (0.03) donc je marque ((oralise en écrivant)) je reconnais les faits (0.02) eu:h (0.02) j'ai commis ces vols (0.03) ca:r§ donc vous m'avez d'abord dit que vous vouliez offrir des cadeaux de Noël,

D.26: à [ma fille

P.3: [à votre fille $=$

D.26: $={ }^{\circ}$ c'est normal $\left[()^{\circ}\right.$

P.3: [donc (> qui a la garde $<$ ) au fait qui: eu:h qui a la garde de votre fille?

D.26: c'est une - une nourrice mai:s je devais l'avoir pour toutes toutes toutes les vacances de de Noël quoi 
P.3: et [c'était prévu ça ?,

D.26: [et je voulais lui faire passer un super Noël

P.3: > voilà< on devait vous la confier ?=

D.26: $=$ (ben) oui.

P.3: > ouais bon ben< et quel âge elle a votre fille ?=

D.26: =elle a sept ans

P.3: sept ans (0.02) ((oralise en écrivant)) car eu:h $\sqrt{ }$ je devais $(0.02)$ recevoir (0.02) euh euh ma fille $(0.01)<$ de sept ans> $(0.01)$ au domicile de mes parents (0.02) au domicile de mes parents où j'habite $(0.05)$ euh elle vit actuellement§ $(0.02)$ placée chez une nourrice ?

D.26: $\left({ }^{\circ}\right)$

P.3: c'est ça ? (0.05) >alors donc< $(($ oralise en écrivant $))$ je voulais de l'argent $(0.03)$ pou:r offrir (0.01) pour lui offrir $(0.01)$ des cadeaux de Noël (0.06) $\sqrt{ }$ en ce moment je craque parce que§ pourquoi ? vou:s - 个alors que: bon d'accord là [vous avez été arrêtée $\uparrow$

D.26: [(je sais pas) ce qui se passe

P.3: $\uparrow$ mai:s euh auparavant $\uparrow$ avant votre arrestation eu:h - >qu'estce qui n'allait pas? alors que vous alliez recevoir votre fille $<$ c'était pas le moment de craquer. $=$

D.26: =non mais [je: justement

P.3: [() d'après ce que vous dites c'est le moment où=

D.26: $=$ on est huit chez nous

P.3: - huit $=$

D.26: [huit

99

P.3: $=\left[\right.$ huit à domi au domicile de vos parents ${ }^{\circ}$ oui $^{\circ}$

100

D.26: e:t huit ça fait beaucoup quand quand tout le monde vous demande de l'argent alors que vous n'avez pas d'argent

101

102

P.3: ${ }^{\circ}$ oui $^{\circ}$ - ((est en train d'écrire $\left.)\right)^{\circ} \mathrm{d}^{\prime}$ accord $^{\circ}$

103

D.26: j'ai j'ai l'impression (que parfois on me tombe) on on me dit d'aller voler !

105

P.3: - ${ }^{\circ} \mathrm{mmh} \mathrm{mmh}^{\circ}$ - e:t chez vous il y il y a vos frères et vos sœurs c'est ça [ou bien

107 D.26: $\left[{ }^{\circ}{ }^{\circ}{ }^{\circ}{ }^{\circ}\right.$

108 P.3: oui d'accord - et depuis juillet vous êtes chez vos parents ?=

109 D.26: =oui 
P.3: (0.03) ((oralise en écrivant)) en ce moment $\$>$ bon je marque< ((oralise en écrivant)) je craque - car eu:h (0.01) nous sommes euh euh - huit - à vivre - chez mes parents§ (0.04) et je ne rapporte aucun salaire?

D.26: [()

P.3: [c'est ce que vous voulez dire ? quand vous le - vous leur pesez quoi ? vous êtes à leur charge - ((oralise en écrivant)) et je suis à leur charge§ (0.02) alors vous m'avez dit aussi euh en arrivant eu:h je prends bon je euh j'ai pas fait >j'ai rien fait (dès) juillet.<

D.26: (0.02) [()

P.3: [vous voulez dire euh vous n'aviez pas volé depuis juillet

D.26: [(mmh mmh) je sais pas

Pr.3: [depuis votre sortie de prison

D.26: ça fait ça fait () depuis quand on m'a arrêté j'ai j'ai volé là et j'aurais j'aurais dû ne pas aller vol vol voler puisque regardez on m'a attrapée ce jour-là

P.3: >oui< ça c'est sûr. (0.03) (alors) ((oralise en écrivant)) je n'avais pas volé (0.03) depuis - ma sortie de prison (0.02) en juillet§ $(0.05) \sqrt{ }$ bien alors là vous alle:z euh voir un avocat si vous voulez bien sûr un avocat commis d'office eu:h vous connaissez donc je suppose gratuit - e:t ensuite vous passez devant le tribunal hein pour être jugée sur ce:s sur ces deux vols (0.01) eu:h <votre: collègue eu:h> (P...) qui a été arrêtée avec vous - était avec vous au départ ?

D.26: - non mais mais elle était pas au courant=

P.3: =elle était pas au courant ?=

D.26: =non

P.3: - mais alors je comprends pas bien eu:h les policiers vous

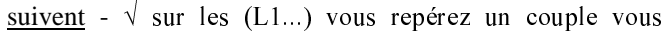
descendez dans la station de métro - euh où a lieu le vol finalement je crois que c'était (L2...),

D.26: mais non mais elle était complètement [(en) dehors

P.3: [et puis vous ressortez le vol commis bien sûr=

D.26: $=$ ouais $=$

P.3: =hein et eu:h - elle vous suit comme ça sans poser de questions?

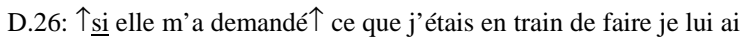


dit - mais t'occupe pas de moi euh ne t'occupe pas de ce que je fais viens et c'est tout quoi

P.3: mmh ouais mais enfin toutes les deux vous êtes connues en prison $\sqrt{ }$ je suppose que vous connaissez toutes les deux le vol [ $\uparrow$ à la tire $\uparrow$ eu:h

D.26: [oui elle a dû elle a dû se douter mais bon ben elle a elle savait pas que que: que moi j'avais pris ce ce portefeuille

P.3: ouais pourquoi vous vous étiez donné rendez-vous ? vous vous étiez donné rendez-vous ou bien [()

D.26: [ouais mai:s le matin parce qu'on voulait passer la journée ensemble

P.3: - ouais - et pourquoi vous êtes allées sur les (L1...) alors ?

D.26: on n'a pas été sur les (L1...) tout de suite

P.3: $[\mathrm{mmh}$

D.26: [on a été à la (L3...) puis à (L4...) et (après) on est venues sur les $(\mathrm{L} 1 \ldots)$

P.3: bon - pour quoi faire?

D.26: pour faire euh mais parce que j'avais un tout petit - un (tout) petit peu d'argent et je voulais acheter un cadeau à ma fille et j'avais pas assez

P.3: ouais - bien alors je vais simplement compléter eu:h - bon ((oralise en écrivant)) j'ai repris eu:h (0.01) repris la consommation - d'héroïne (0.04) depuis une semaine§ (0.02) ((signe)) bien donc vous allez voir l'avocat et ensuite vous passez devant le tribunal. - > voilà < [je vous demande de signer

D.26: [() le tribunal et tout ()

P.3: pardon

D.26: je dois [passer ()

P.3: [à quelle heure ? après le temps de voir l'avocat quoi d'ici une heure une heure trente je ne sais pas bien hein ((donne un petit coup sur la table)) voilà je vous demande une signature là en bas

D.26: $(0.06)(($ signe $))($ voilà $)=$

P.3: $=$ merci

(0.08) ((P.3 prépare le dossier, D.26 se lève et est menottée $))$

(G.): ${ }^{\circ}$ merci bien $^{\circ}$

(P.3): ${ }^{\circ} \mathrm{merci}^{\circ}$

186 (0.07) ((D.26 et G. sortent $))$ 
ATKINSON J. M., HERITAGE J. (eds.) (1984), Structures of social action. Studies in conversational analysis, Cambridge, Cambridge University press.

BACCUS M. D. (1986), "Sociological indication and the visibility criterion of real world social theorizing", in Garfinkel H. (ed.), Ethnomethodological studies of work, London, Routledge and Kegan Paul, p. 1-19.

CARLIN A. P. (2003), "Pro forma arrangements. The visual availability of textual artefacts", Visual studies, vol. 18, n 1, p. 6-20.

CICOUREL A. V. (1975), "Discourse and text. Cognitive and linguistic processes in studies of social structure", Versus, vol. 12, p. 33-84.

CONEIN B. (1987), «Quelques formes de l'interprétation de la conversation dans la conversation », Lexique, ${ }^{\circ}$ 5, p. 37-64.

COULTER J. (1990), "Elementary properties of argument sequences", in Psathas G. (ed.), Interaction competence, Lanham, University press of America, p. 181-203.

CUFF E. C., FRANCIS D. W. (1978), "Some features of 'invited stories' about marriage breakdown", International journal of the sociology of language, $\mathrm{n}^{\circ} 18$, p. 111-133.

EGLIN P., WIDEMAN D. (1986), "Inequality in professional service encounters. Verbal strategies of control versus task performance in calls to the police", Zeitschrift für Soziologie, vol. 15, n 5, p. 341-362.

GARFINKEL H. (1984) [1967], Studies in ethnomethodology, Cambridge, Polity press.

GARFINKEL H. (2001) [1996], «Le programme de l'ethnomethodologie », in Fornel M. de, Ogien A., Quéré L. (eds.), L'ethnométhodologie. Une sociologie radicale, Paris, La découverte, p. 33-56.

GARFINKEL H. (2002), Ethnomethodology's program. Working out Durkheim's aphorism, Lanham, Rowman and Littlefield.

GARFINKEL H., LYNCH M., LIVINGSTON E. (1981), "The work of a discovering science construed with materials from the optically discovered pulsar", Philosophy of the social sciences, vol. 11, $\mathrm{n}^{\circ} 2$, p. 131-158.

GARFINKEL H., SACKS H. (1986 [1969]), "On formal structures of practical actions", in Garfinkel H. (ed.), Ethnomethodological studies of work, London, Routledge and Kegan Paul, p. 160-193.

GARFINKEL H. et WIEDER D. L. (1992), "Two incommensurable, asymmetrically alternate technologies of social analysis", in Watson G. et Seiler R. M. (eds.), Text in context. Contributions to ethnomethodology, London, Sage, p. 175-206. 
GONZÁLEZ MARTÍNEZ E. (2001), «Comment agir en confiance avec un partenaire dont on se méfie ? », Réseaux, vol. 19, n 108, p. 87-123.

GONZÁLEZ MARTÍNEZ E. (2003), L'audition de comparution immédiate. L'organisation des échanges langagiers, Thèse de doctorat en sciences socialessociologie, Université de Lausanne-EHESS.

GOODWIN C. (1994), "Professional vision", American anthropologist, vol. 96, $\mathrm{n}^{\circ} 3$, p. 606-633.

GOODWIN C. et GOODWIN M. H. (1997), « La coopération au travail dans un aéroport », Réseaux, $\mathrm{n}^{\circ} 85$, p. 129-162.

GOODWIN M. H., GOODWIN C. (1987), "Children's arguing”, in Philips S. U., Steele S. et Tanz C. (eds.), Language, gender and sex in comparative perspective, Cambridge, Cambridge University press, p. 200-248.

GRUBER H. (1998), "Disagreeing. Sequential placement and internal structure of disagreements in conflict episodes", Text, vol. 18, $\mathrm{n}^{\circ} 4$, p. 467-503.

HAVE P. ten (1991), "Talk and institutions. A reconsideration of the 'asymmetry' of doctor-patient interaction", in Boden D. et Zimmerman D. H. (eds.), Talk and social structure. Studies in ethnomethodology and conversation analysis, Polity press, Cambridge, p. 138-163.

HEATH C., LUFF P. (1996), "Convergent activities. Line control and passenger information on the London underground", in Engeström Y. et Middleton D. (eds.), Cognition and communication at work, Cambridge, Cambridge University press, p. 96-129.

HERITAGE J., ROTH A. (1995), "Grammar and institution. Questions and questioning in the broadcast news interview", Research on language and social interaction, vol. $28, \mathrm{n}^{\circ} 1$, p. 1-60.

HESTER S., FRANCIS D. (2003), “Analysing visually available mundane order. A walk to the supermarket", Visual studies, vol. 18, n 1 , p. 36-46.

HUTCHBY I. (1996), Confrontation talk. Arguments, asymmetries and power on talk radio, Mahwah, Lawrence Erlbaum.

JEFFERSON G. (1978), "Sequential aspects of storytelling in conversation", in Schenkein J. (ed.), Studies in the organization of conversational interaction, New York, Academic press, p. 219-248.

JEFFERSON G. (1988), "On the sequential organisation of troubles-talk in ordinary conversation", Social problems, vol. 35 , n 4, p. 418-441.

JEFFERSON G., LEE J. R. E. (1992) [1981], "The rejection of advice. Managing the problematic convergence of a 'troubles-telling' and a 'service encounter", in 
Drew P. et Heritage J. (eds.), Talk at work. Interaction in institutional settings, Cambridge, Cambridge University press, p. 521-548.

KOMTER M. L. (2001), «La construction de la preuve dans un interrogatoire de police », Droit et société, $\mathrm{n}^{\circ} 48$, p. 367-393.

LEE J. R. E., WATSON R. (1992-1993), « Regards et habitudes des passants. Les arrangements de visibilité de la locomotion», Les annales de la recherche urbaine, $\mathrm{n}^{\circ}$ 57-58, p. 101-109.

LYNCH M. (1982), "Closure and disclosure in pre-trial arguments", Human studies, vol. $5, \mathrm{n}^{\circ} 4$, p. 285-318.

LYNCH M. (1985), "Discipline and the material form of images. An analysis of scientific visibility", Social studies of science, vol. 15, $\mathrm{n}^{\circ}$ 1, p. 37-66.

MAYNARD D. W. (1985), "How children start arguments", Language in society, vol. $14, \mathrm{n}^{\circ} 1$, p. $1-29$

MAYNARD D. W. (1991), "Interaction and asymmetry in clinical discourse", American journal of sociology, vol. 97, $\mathrm{n}^{\circ} 2$, p. 448-495.

MCHOUL A. W. (1987), "Why there are no guarantees for interrogators", Journal of pragmatics, vol. 11, p. 455-471.

MERRITT M. (1976), "On questions following questions in service encounters", Language in society, vol. 5, $\mathrm{n}^{\circ} 3$, p. 315-357.

POLLNER M. (1979), "Explicative transactions. Making and managing meaning in traffic court", in Psathas G. (ed.), Everyday language. Studies in ethnomethodology, New York, Irvington, p. 227-255.

PSATHAS G. (1990), "The organization of talk, gaze and activity in a medical interview", in Psathas G. (ed.), Interaction competence, Lanham, University press of America, p. 205-230.

QUERE L. (1985), «L'argument sociologique de Garfinkel», Problèmes d'épistémologie en sciences sociales, $\mathrm{n}^{\circ} 3$, p. 100-137.

QUERE L. (1999), «Action située et perception du sens », in Fornel M. de et Quéré L. (eds.), La logique des situations. Nouveaux regards sur l'écologie des activités sociales (Raisons pratiques $n^{\circ} 10$ ), Paris, Editions EHESS, p. 301-338.

QUERE L., BREZGER D. (1992-1993), «L'étrangeté mutuelle des passants. Le mode de coexistence du public urbain ", Les annales de la recherche urbaine, $\mathrm{n}^{\circ}$ 5758 , p. $89-100$.

RELIEU M., QUERE L. (1998), «Mobilité, perception et sécurité dans les espaces publics urbains. Comment se déplacer quand on ne voit plus les choses comme «tout le monde" ?», in Ansidei H. et al. (eds.), Les risques urbains. Acteurs, systèmes de prévention, Paris, Anthropos, p. 91-120. 
RYAVE A. L. (1978), "On the achievement of a series of stories", in Schenkein J. (ed.), Studies in the organization of conversational interaction, New York, Academic press, p. 113-132.

RYAVE A. L., SCHENKEIN J. N. (1974), "Notes on the art of walking", in Turner R. (ed.), Ethnomethodology. Selected readings, Harmondsworth, Penguin books, p. $265-274$

SACKS H. (1972), "Notes on police assessment of moral character", in Sudnow D. (ed.), Studies in social interaction, New York, The free press, p. 280-293.

SACKS H. (1974), "An analysis of the course of a joke's telling in conversation", in Bauman R. et Sherzer J. (eds.), Explorations in the ethnography of speaking, Cambridge, Cambridge University press, p. 337-353.

SACKS H. (1978), "Some technical considerations of a dirty joke", in Schenkein J. (ed.), Studies in the organization of conversational interaction, New York, Academic press, p. 249-269.

SACKS H. (1984), "On doing 'being ordinary", in Atkinson J. M. et Heritage J. (eds.), Structures of social action. Studies in conversational analysis, Cambridge, Cambridge University press, p. 413-429.

SACKS H. (1987), "On the preferences for agreement and contiguity in sequences in conversation", in Button G. et Lee J. R. E. (eds.), Talk and social organisation, Clevedon, Multilingual matters, p. 54-69.

SACKS H. (1989a), "Lecture eleven. On exchanging glances", Human studies, vol. $12, \mathrm{n}^{\circ} 3-4$, p. 333-348.

SACKS H. (1989b), "Lecture fourteen. The inference-making machine", Human studies, vol. $12, \mathrm{n}^{\circ}$ 3-4, p. 379-393.

SACKS H. (1992), Lectures on conversation. I-II, Oxford, Blackwell.

SCHEGLOFF E. A., SACKS H. (1973), "Opening up closings", Semiotica, vol. 8, $\mathrm{n}^{\circ} 4$, p. 289-327.

SCHEGLOFF E. A. (1988/1989), "From interview to confrontation. Observations of the Bush/Rather encounter", Research on language and social interaction, vol. 22, p. $215-240$.

SCHEGLOFF E. A. (1990) [1968], "Sequencing in conversational openings", in Coulter J. (ed.), Ethnomethodological sociology, Aldershot, Edward Elgar, p. 254-352.

SHARROCK W., WATSON D. R. (1990), «L'unité du faire et du dire. L'action et l'organisation sociales comme phénomènes observables et descriptibles », in Pharo P. et Quéré L. (eds.), Les formes de l'action, Paris, Editions EHESS, p. 227-254.

STEFANI G., LEVASSEUR G., BOULOC B. (1993) [1964], Procédure pénale, Paris, Dalloz. 
SUDNOW D. (1972), "Temporal parameters of interpersonal observation", in Sudnow D. (ed.), Studies in social interaction, New York, The free press, p. 259-279.

WHALEN J., ZIMMERMAN D. H., WHALEN M. R. (1992a), « Une conversation fatale », Réseaux, $\mathrm{n}^{\circ} 55$, p. 145-178.

ZIMMERMAN D. H. (1992b), "The interactional organization of calls for emergency assistance", in Drew P. et Heritage J. (eds.), Talk at work. Interaction in institutional settings, Cambridge, Cambridge University press, p. 418-469. 